\title{
Brave New World
}

In 1911, exactly 100 years ago, Hirschel ${ }^{1}$ published his observations on axillary brachial plexus block, obtaining anesthesia of the arm. The local anesthetic used was novocaine and, based on the relatively low toxicity of this agent, large volumes $(30-40 \mathrm{~mL})$ of a $2.0 \%$ solution were used.

In the same year and shortly after this first communication, Kulenkampff published his works on supraclavicular brachial plexus block, at the place it slides over the first rib ${ }^{2}$. He also used $2.0 \%$ novocaine solution, but with adrenaline, obtaining anesthesia for surgeries in the arm with smaller volumes, approximately $10 \mathrm{~mL}$. Kulenkampff technique included an important anatomical reference point for orientation of the needle, ie, the first rib.

But these brachial plexus block techniques were almost primitive, based on anatomical orientations and strategies proposed by the authors whose techniques were named after. The incidence of failures could not be ignored and they also carried the risk of important complications, such as pneumothorax in Kulenkampff technique ${ }^{3}$. Paresthesia indicated the proximity of the needle to nerve structures. The adiposity of the supraclavicular fossa represented an obstacle, which required the needle to penetrate deeper until it hit the first rib.

In 1964, Winnie and Collins ${ }^{4}$ published their experience with the subclavian perivascular brachial plexus block technique. It is a modification of the original Kulenkampff technique, using the needle in the middle of the clavicle in a perpendicular (and not medial) direction to the first rib. In the absence of paresthesia, the subclavian artery could be used as an important reference point. The local anesthetic used was lidocaine, and $40 \mathrm{~mL}$ of $1.0 \%$ solution produced satisfactory anesthesia. But the risk of pneumothorax was still present.

In 1970, Winnie ${ }^{5}$ described the interscalene brachial plexus block emphasizing the importance of the muscle sheath that involves the origins of the plexus in the neck. The lateral border of the sternocleidomastoid muscle is an important anatomical reference, as well as the sulcus between the anterior and middle scalene muscles. The needle should be in a caudal direction, since the medial direction allows it to go through the intervertebral foramen and consequent epidural, subarachnoid, or intra-arterial injection of the local anesthetic. The volume of $25-30 \mathrm{~mL}$ of the solution produces adequate analgesia; larger volumes allow for more extensive block- ades, up to the cervical plexus. Horner's syndrome is not uncommon due to cervical sympathetic blockade.

In 1993, Ganta et al. ${ }^{6}$ described the use of transcutaneous nerve stimulation to localize the nerve in interscalene block. This represented a great advance, with substitution of invasive nerve stimulation through a needle by a non-invasive method to localize the structure to be blocked. Soon after, studies on its use in several types of peripheral nerve blocks multiplied ${ }^{7-9}$ and it lead to the creation of a commercially available device, stimuplex, for cutaneous identification of the brachial plexus ${ }^{10}$.

Although the use of ultrasound in regional anesthesia had been described for the first time in $1978{ }^{11}$, it was only in the last decade, due to great advances in resolution of ultrasound images of nerve roots, peripheral nerves, and fasciae, that this method was disseminated, and, currently, the brachial plexus block is one of its most common applications ${ }^{12-16}$. The reduction in the size of ultrasound equipment and its transformation in portable devices with elevated resolution power and image quality have propagated the method in several locations in which anesthetic blocks are performed, especially brachial plexus.

The advantages are patent. Studies published in 2011 have demonstrated that: a) ultrasound is a much more accurate method than transcutaneous electric nerve stimulation to identify the brachial plexus in the neck 17 ; b) ultrasound decreases the minimal effective volume of the local anesthetic solution ( $0.5 \%$ ropivacaína) by 6 -fold when compared with transcutaneous electric nerve stimulation in interscalene brachial plexus block ${ }^{18}$.

The benefits regarding accuracy, quality, and safety are evident.

One hundred years later, we can only say one thing, paraphrasing Aldous Huxley ${ }^{19}$ : "the world of modern anesthesia is a brave new world."

José Roberto Nociti, TSA-SBA

Member of the Editorial Board

Revista Brasileira de Anestesiologia

Responsible for the CET-SBA of the Santa Casa de Misericórdia de Ribeirão Preto 